\title{
Algebraic relations between special values of multiple sine functions
}

by

Hidekazu TANAKa (Tokyo)

1. Introduction. The algebraicity of a product of division values of the double sine function $S_{2}(\alpha,(1, \tau))$ at $\alpha \in \mathbb{Q}+\mathbb{Q} \tau$ (see [KW], [S], [Tang]) is very interesting from the viewpoint of Kronecker's Jugendtraum for a real quadratic field. We expect the similar situation for division values of multiple sine functions. In this paper, we study algebraic relations between special values of multiple sine functions. Recall that the multiple sine function $S_{r}\left(x,\left(\omega_{1}, \ldots, \omega_{r}\right)\right)$ is defined by

$S_{r}\left(x,\left(\omega_{1}, \ldots, \omega_{r}\right)\right)$

$=\prod_{n_{1}, \ldots, n_{r} \geq 0}\left(n_{1} \omega_{1}+\cdots+n_{r} \omega_{r}+x\right)\left(\prod_{n_{1}, \ldots, n_{r} \geq 1}\left(n_{1} \omega_{1}+\cdots+n_{r} \omega_{r}-x\right)\right)^{(-1)^{r-1}}$,

where $\prod$ denotes the regularized product of Deninger $[\mathrm{D}]$ :

$$
\prod_{\lambda \in \Lambda} \lambda=\exp \left(-\left.\frac{d}{d s} \sum_{\lambda \in \Lambda} \lambda^{-s}\right|_{s=0}\right) .
$$

We refer to $[\mathrm{KoKu}],[\mathrm{KuKo}],[\mathrm{KW}]$ for the details of the theory of multiple sine functions. We write $S_{r}(x,(1, \ldots, 1))=S_{r}(x)$ for simplicity.

Theorem 1. Let $m$ be a positive integer. Then

$$
\prod_{k=0}^{m-1} S_{2 m-k}(1)^{(-1)^{k}\left(\begin{array}{c}
m-1 \\
k
\end{array}\right)}=1 .
$$

THEOREM 2. Let

$$
\delta(r):= \begin{cases}1 & \text { if } r \text { is odd }, \\ 0 & \text { if } r \text { is even } .\end{cases}
$$

2000 Mathematics Subject Classification: Primary 11M36.

Key words and phrases: multiple sine function, algebraicity, transcendence. 
Then

$$
\begin{aligned}
\prod_{k=0}^{[r / 2]-1} S_{r-k}(1)^{(-1)^{k}} & \sum_{l=1+k}^{[r / 2]}\left(\begin{array}{c}
r \\
2 l
\end{array}\right)\left(\begin{array}{c}
l-1 \\
k
\end{array}\right) \\
& \times \prod_{k=0}^{[r / 2]+\delta(r)-1} S_{r-k}(1 / 2)^{(-1)^{k} \sum_{l=1+k}^{[r / 2]+\delta(r)}\left(\begin{array}{c}
r \\
2 l-1
\end{array}\right)\left(\begin{array}{c}
l-1 \\
k
\end{array}\right)=2 .}
\end{aligned}
$$

THEOREM 3.

$$
\begin{aligned}
& S_{3}(1,(1,1,1 / 2))^{-8} S_{3}(1)^{2}=2, \\
& S_{4}(1,(1,1,1,1 / 2))^{-16} S_{4}(1)^{10}=2, \\
& S_{5}(1,(1,1,1,1,1 / 2))^{-128} S_{5}(1)^{8} S_{4}(1)^{82}=32, \\
& S_{6}(1,(1,1,1,1,1,1 / 2))^{-256} S_{6}(1)^{-38} S_{5}(1)^{228}=128 .
\end{aligned}
$$

Remark 1.1. Kurokawa and Koyama [KuKo, Lemma 3.2] calculated $S_{r}(1)$. For example, we have

$$
\begin{aligned}
& S_{3}(1)=S_{4}(1)=\exp \left(\frac{\zeta(3)}{4 \pi^{2}}\right), \\
& S_{5}(1)=\exp \left(\frac{11}{48 \pi^{2}} \zeta(3)-\frac{\zeta(5)}{16 \pi^{4}}\right), \\
& S_{6}(1)=\exp \left(\frac{5}{24 \pi^{2}} \zeta(3)-\frac{\zeta(5)}{8 \pi^{4}}\right) .
\end{aligned}
$$

Moreover, the value $S_{r}(\alpha)$ at $\alpha \in \mathbb{Q}-\mathbb{Z}$ was obtained in [Tana, Theorem 1.1].

Set $\underline{\omega}_{k}:=(\overbrace{\omega, \ldots, \omega}^{k})$ for a positive integer $k$.

TheOREM 4. Let $m$ be a positive integer. Then

$$
S_{2 m}\left(m,\left(\underline{1}_{m}, \underline{\tau}_{m}\right)\right)=S_{2 m}\left(m,\left(\underline{1}_{m}, \underline{\tau}_{m}^{-1}\right)\right)^{-1} .
$$

Remark 1.2. Put $m=1$ in Theorem 4 . Then

$$
S_{2}(1,(1, \tau))=S_{2}\left(1,\left(1, \tau^{-1}\right)\right)^{-1} .
$$

In fact, following Koyama, Kurokawa, Tangedal and Wakayama (see [KoKu, (4) or (5) of Theorem 1], [KW, Lemma 2.2], [Tang, Lemma 6] for details) we have

$$
S_{2}(1,(1, \tau))=\sqrt{\tau}
$$

TheOREm 5. Let $r \geq 2$ be an integer and $\tau>0$ be algebraic irrational.

(1) One of the numbers $S_{r}\left(t+1,\left(\underline{1}_{r-1}, \tau\right)\right), t=1, \ldots, r-1$, is transcendental.

(2) One of the numbers $S_{r}\left((t+1) \tau+1 / 2,\left(\underline{1}_{r-1}, \tau\right)\right), t=1, \ldots, r-1$, is transcendental. 
(3) One of the numbers $\prod_{k=1}^{N-1} S_{r}\left(t+k / N,\left(\underline{1}_{r-1}, \tau\right)\right), t=1, \ldots, r-1$, is transcendental. In particular, one of the numbers $S_{r}((2 t+1) / 2$, $\left.\left(\underline{1}_{r-1}, \tau\right)\right), t=1, \ldots, r-1$, is transcendental.

(4) Let $\delta=1, \tau$. Then one of the numbers $S_{r}\left((1+\tau) / 2+t \delta,\left(\underline{1}_{r-1}, \tau\right)\right)$, $t=1, \ldots, r-1$, is transcendental.

REMARK 1.3. When $r=2$, Theorem 5(1) is a result of Kurokawa and Wakayama [KW, Theorem 1.2].

THEOREM 6.

(1) Let $n \geq 2$ be an integer. When $\tau>0$ is algebraic irrational, the number $S_{2}(1 / 2+n \tau,(1, \tau))$ is transcendental.

(2) Let $n \geq 1$ and $N \geq 2$ be integers. When $\tau>0$ is algebraic irrational, the number $\prod_{k=1}^{N-1} S_{2}(k / N+n,(1, \tau))$ is transcendental. In particular, $S_{2}(1 / 2+n,(1, \tau))$ is transcendental.

TheOREM 7. Let $n_{1}, n_{2}$ be non-negative integers, not both zero. When $\tau>0$ is real quadratic irrational, $S_{2}\left((1+\tau) / 2+n_{1}+n_{2} \tau,(1, \tau)\right)$ is transcendental. When $n_{1}=0$ or $n_{2}=0$, this holds for any algebraic irrational $\tau>0$.

THEOREM 8. When $\tau>0$ is rational, the values in Theorems 6 and 7 are algebraic.

Theorem 1.2 of $[\mathrm{KW}]$ is shown via the Gelfond-Schneider theorem (see [Bak, Theorem 2.1]). In the same way we prove Theorems 6 and 7 .

\section{Proofs of Theorems 1-4}

Proof of Theorem 1. First we show that for any non-negative integer $k$,

$$
S_{r}(x)=\prod_{l=0}^{k} S_{r-l}(x-k)^{(-1)^{l}\left(\begin{array}{c}
k \\
l
\end{array}\right) .}
$$

We recall the quasi-periodicity of multiple sine functions (see $[\mathrm{KuKo}]$ for details):

Lemma 2.1 ([KuKo, Theorem 2.1(a)]). For $j=1, \ldots, r$,

$$
S_{r}\left(x+\omega_{j},\left(\omega_{1}, \ldots, \omega_{r}\right)\right)=\frac{S_{r}\left(x,\left(\omega_{1}, \ldots, \omega_{r}\right)\right)}{S_{r-1}\left(x,\left(\omega_{1}, \ldots, \omega_{j-1}, \omega_{j+1}, \ldots, \omega_{r}\right)\right)},
$$

where we put $S_{0}(x, \cdot)=-1$.

When $k=1$, from (2.2) we have

$$
S_{r}(x)=\frac{S_{r}(x-1)}{S_{r-1}(x-1)} .
$$


Inductively suppose (2.1) holds for $k$. Then using (2.2) we have

$$
\begin{aligned}
S_{r}(x)= & \prod_{l=0}^{k}\left\{S_{r-l}(x-(k+1)) S_{r-l-1}(x-(k+1))^{-1}\right\}^{(-1)^{l}\left(\begin{array}{c}
k \\
l
\end{array}\right)} \\
= & S_{r}(x-(k+1)) S_{r-(k+1)}(x-(k+1))^{(-1)^{k+1}} \\
& \times \prod_{l=1}^{k} S_{r-l}(x-(k+1))^{(-1)^{l}\left(\begin{array}{c}
k \\
l
\end{array}\right)} \\
& \times \prod_{l=0}^{k-1} S_{r-l-1}(x-(k+1))^{(-1)^{l+1}\left(\begin{array}{c}
k \\
l
\end{array}\right)} \\
= & S_{r}(x-(k+1)) S_{r-(k+1)}(x-(k+1))^{(-1)^{k+1}} \\
& \times \prod_{l=1}^{k} S_{r-l}(x-(k+1))^{(-1)^{l}\left\{\left(\begin{array}{l}
k \\
l
\end{array}\right)+\left(\begin{array}{c}
k \\
l-1
\end{array}\right)\right\}} \\
= & \prod_{l=0}^{k+1} S_{r-l}(x-(k+1))^{(-1)^{l}\left(\begin{array}{c}
k+1 \\
l
\end{array}\right) .}
\end{aligned}
$$

Hence we obtain (2.1). Put $r=2 m, x=m$ and $k=m-1$ in (2.1) to obtain

$$
S_{2 m}(m)=\prod_{l=0}^{m-1} S_{2 m-l}(1)^{(-1)^{l}\left(\begin{array}{c}
m-1 \\
l
\end{array}\right)} .
$$

By the definition of the multiple sine function, we see that

$$
S_{2 m}(m)=\frac{\prod_{n_{1}, \ldots, n_{2 m} \geq 0}\left(n_{1}+\cdots+n_{2 m}+m\right)}{\prod_{n_{1}, \ldots, n_{2 m} \geq 0}\left(n_{1}+\cdots+n_{2 m}+m\right)}=1 .
$$

Hence, Theorem 1 is proved.

Proof of Theorem 2. First, we recall a lemma.

Lemma 2.2 ([KuKo, Theorem 2.1(c)]). Let $N \geq 2$ be an integer. Then

$$
\prod_{\substack{k_{1}, \ldots, k_{r}=0 \\\left(k_{1}, \ldots, k_{r}\right) \neq(0, \ldots, 0)}}^{N-1} S_{r}\left(\frac{k_{1} \omega_{1}+\cdots+k_{r} \omega_{r}}{N},\left(\omega_{1}, \ldots, \omega_{r}\right)\right)=N .
$$

Putting $N=2$ and $\omega_{1}=\cdots=\omega_{r}=1$ in $(2.3)$, we get

$$
\prod_{k=1}^{r} S_{r}(k / 2)^{\left(\begin{array}{l}
r \\
k
\end{array}\right)}=2 \text {. }
$$


Therefore,

$$
\prod_{l=1}^{[r / 2]} S_{r}(l)^{\left(\begin{array}{c}
r \\
2 l
\end{array}\right)} \prod_{l=1}^{[r / 2]+\delta(r)} S_{r}(l-1 / 2)^{\left(\begin{array}{c}
r \\
2 l-1
\end{array}\right)}=2 .
$$

From (2.1) we have

$$
\begin{aligned}
S_{r}(l) & =\prod_{k=0}^{l-1} S_{r-k}(1)^{(-1)^{k}\left(\begin{array}{c}
l-1 \\
k
\end{array}\right),} \\
S_{r}(l-1 / 2) & =\prod_{k=0}^{l-1} S_{r-k}(1 / 2)^{(-1)^{k}\left(\begin{array}{c}
l-1 \\
k
\end{array}\right) .}
\end{aligned}
$$

Hence,

$$
\prod_{l=1}^{[r / 2]} \prod_{k=0}^{l-1} S_{r-k}(1)^{(-1)^{k}}\left(\begin{array}{c}
r \\
2 l
\end{array}\right)\left(\begin{array}{c}
l-1 \\
k
\end{array}\right) \prod_{l=1}^{[r / 2]+\delta(r)} \prod_{k=0}^{l-1} S_{r-k}(1 / 2)^{(-1)^{k}\left(\begin{array}{c}
r \\
2 l-1
\end{array}\right)\left(\begin{array}{c}
l-1 \\
k
\end{array}\right)}=2 .
$$

This formula can be rewritten to give the formula of Theorem 2 .

ExAMPLE 2.1 (Application of Theorem 2).

$$
\begin{aligned}
& S_{2}(1 / 2)^{2}=2, \\
& S_{3}(1)^{3} S_{3}(1 / 2)^{4} S_{2}(1 / 2)^{-1}=2, \\
& S_{4}(1)^{6} S_{4}(1 / 2)^{8} S_{3}(1 / 2)^{-4}=2, \\
& S_{5}(1)^{15} S_{4}(1)^{-5} S_{5}(1 / 2)^{16} S_{4}(1 / 2)^{-12} S_{3}(1 / 2)=2, \\
& S_{6}(1)^{30} S_{5}(1)^{-15} S_{6}(1 / 2)^{32} S_{5}(1 / 2)^{-32} S_{4}(1 / 2)^{6}=2 .
\end{aligned}
$$

Proof. Putting $r=3,5$ in Theorem 2, we obtain (2) and (4) immediately. Putting $r=2$ in Theorem 2 yields

$$
S_{2}(1) S_{2}(1 / 2)^{2}=2,
$$

and putting $m=1$ in Theorem 1 gives

$$
S_{2}(1)=1 \text {. }
$$

Hence, (1) is proved. Similarly, put $r=4$ and $r=6$ in Theorem 2 to get,

$$
\begin{aligned}
& S_{4}(1)^{7} S_{3}(1)^{-1} S_{4}(1 / 2)^{8} S_{3}(1 / 2)^{-4}=2, \\
& S_{6}(1)^{31} S_{5}(1)^{-17} S_{4}(1) S_{6}(1 / 2)^{32} S_{5}(1 / 2)^{-32} S_{4}(1 / 2)^{6}=2 .
\end{aligned}
$$

Putting $m=2$ and $m=3$ in Theorem 1, we see that

$$
\begin{aligned}
& S_{4}(1) S_{3}(1)^{-1}=1, \\
& S_{6}(1) S_{5}(1)^{-2} S_{4}(1)=1 .
\end{aligned}
$$

This yields (3) and (5).

Proof of Theorem 3. We prepare a lemma. 
Lemma 2.3. Let $l, N$ be positive integers. Then

$$
S_{r}\left(x,\left(\underline{1}_{r-1}, l / N\right)\right)=\prod_{k=0}^{N-1} S_{r}\left(x+l k / N,\left(\underline{1}_{r-1}, l\right)\right) .
$$

Proof of Lemma 2.3. Using

$$
\begin{aligned}
\zeta_{r}\left(s, x,\left(\underline{1}_{r-1}, l / N\right)\right): & \sum_{\substack{n_{1}, \ldots, n_{r} \geq 0 \\
N-1}}\left(x+n_{1}+\cdots+n_{r-1}+l n_{r} / N\right)^{-s} \\
& =\sum_{k=0}^{N} \sum_{n_{1}, \ldots, n_{r} \geq 0}\left(x+l k / N+n_{1}+\cdots+n_{r-1}+l n_{r}\right)^{-s},
\end{aligned}
$$

we have

$$
\begin{aligned}
\prod_{n_{1}, \ldots, n_{r} \geq 0}\left(n_{1}+\cdots+\right. & \left.n_{r-1}+\ln _{r} / N+x\right)^{-1} \\
& =\prod_{k=0}^{N-1} \prod_{n_{1}, \ldots, n_{r} \geq 0}\left(n_{1}+\cdots+n_{r-1}+l n_{r}+x+l k / N\right)^{-1} .
\end{aligned}
$$

From

$$
\begin{aligned}
\prod_{n_{1}, \ldots, n_{r} \geq 1} & \left(n_{1}+\cdots+n_{r-1}+\ln _{r} / N-x\right)^{-1} \\
= & \prod_{n_{1}, \ldots, n_{r} \geq 0}\left(n_{1}+\cdots+n_{r-1}+l n_{r} / N+r-1-x+l / N\right)^{-1} \\
= & \prod_{k=0}^{N-1} \prod_{n_{1}, \ldots, n_{r} \geq 0}\left(n_{1}+\cdots+n_{r-1}+l n_{r}+r-1-x+l(k+1) / N\right)^{-1} \\
= & \prod_{k=0}^{N-1} \prod_{n_{1}, \ldots, n_{r} \geq 0}\left(n_{1}+\cdots+n_{r-1}+l n_{r}+r-1-x+l(N-k) / N\right)^{-1} \\
= & \prod_{k=0}^{N-1} \prod_{n_{1}, \ldots, n_{r} \geq 1}\left(n_{1}+\cdots+n_{r-1}+l n_{r}-x-l k / N\right)^{-1}
\end{aligned}
$$

we obtain Lemma 2.3 .

Put $x=1, l=1$ and $N=2$ in Lemma 2.3 to obtain

$$
S_{r}\left(1,\left(\underline{1}_{r-1}, 1 / 2\right)\right)=S_{r}(1) S_{r}(3 / 2)=S_{r}(1) S_{r}(1 / 2) S_{r-1}(1 / 2)^{-1},
$$

where we used the quasi-periodicity of multiple sine functions:

$$
S_{r}(3 / 2)=S_{r}(1 / 2) S_{r-1}(1 / 2)^{-1} .
$$


Now we show (1) of Theorem 3. When $r=3$, from (2.6) and Example $2.1(2)$ we see that

$$
S_{3}(1,(1,1,1 / 2))=S_{3}(1) S_{2}(1 / 2)^{-1}\left\{2 S_{3}(1)^{-3} S_{2}(1 / 2)\right\}^{1 / 4} .
$$

Since (see Example 2.1(1))

we obtain (1) of Theorem 3 .

$$
S_{2}(1 / 2)=\sqrt{2}
$$

Next we show (2) of Theorem 3. When $r=4$, from (2.6) and Example 2.1(3) we see that

$$
S_{4}(1,(1,1,1,1 / 2))=S_{4}(1) S_{3}(1 / 2)^{-1}\left\{2 S_{4}(1)^{-6} S_{3}(1 / 2)^{4}\right\}^{1 / 8} .
$$

Hence, using Example 2.1(2) we have

$$
S_{4}(1,(1,1,1,1 / 2))^{8}=2 S_{4}(1)^{2} S_{3}(1 / 2)^{-4}=2 S_{4}(1)^{2}\left\{2 S_{3}(1)^{-3} S_{2}(1 / 2)\right\}^{-1} \text {. }
$$

Applying (2.4) we obtain (2) of Theorem 3.

Next we show (3) of Theorem 3. From (2.6) for $r=5$ and Example 2.1(4) we see that

$$
\begin{aligned}
& S_{5}(1,(1,1,1,1,1 / 2)) \\
& \quad=S_{5}(1) S_{4}(1 / 2)^{-1}\left\{2 S_{5}(1)^{-15} S_{4}(1)^{5} S_{4}(1 / 2)^{12} S_{3}(1 / 2)^{-1}\right\}^{1 / 16} .
\end{aligned}
$$

Hence, using Example 2.1(2) \& (3) we have

$$
\begin{aligned}
S_{5}(1,(1,1,1,1,1 / 2))^{16} & =2 S_{5}(1) S_{4}(1)^{5} S_{4}(1 / 2)^{-4} S_{3}(1 / 2)^{-1} \\
& =2 S_{5}(1) S_{4}(1)^{5} S_{3}(1 / 2)^{-1}\left\{2 S_{4}(1)^{-6} S_{3}(1 / 2)^{4}\right\}^{-1 / 2} \\
& =2^{1 / 2} S_{5}(1) S_{4}(1)^{8} S_{3}(1 / 2)^{-3} \\
& =2^{1 / 2} S_{5}(1) S_{4}(1)^{8}\left\{2 S_{3}(1)^{-3} S_{2}(1 / 2)\right\}^{-3 / 4} \\
& =2^{-5 / 8} S_{5}(1) S_{4}(1)^{8} S_{3}(1)^{9 / 4} .
\end{aligned}
$$

Applying (2.4) we obtain (3) of Theorem 3.

Finally, we show (4) of Theorem 3. From (2.6) for $r=6$ and Example 2.1(5) we see that

$$
\begin{aligned}
& S_{6}(1,(1,1,1,1,1,1 / 2)) \\
& \quad=S_{6}(1) S_{5}(1 / 2)^{-1}\left\{2 S_{6}(1)^{-30} S_{5}(1)^{15} S_{5}(1 / 2)^{32} S_{4}(1 / 2)^{-6}\right\}^{1 / 32} .
\end{aligned}
$$

Hence, using Example 2.1(2) \& (3) we have

$$
\begin{aligned}
S_{6}(1,(1,1,1,1,1,1 / 2))^{32} & =2 S_{6}(1)^{2} S_{5}(1)^{15} S_{4}(1 / 2)^{-6} \\
& =2 S_{6}(1)^{2} S_{5}(1)^{15}\left\{2 S_{4}(1)^{-6} S_{3}(1 / 2)^{4}\right\}^{-3 / 4} \\
& =2^{1 / 4} S_{6}(1)^{2} S_{5}(1)^{15} S_{4}(1)^{9 / 2} S_{3}(1 / 2)^{-3} \\
& =2^{1 / 4} S_{6}(1)^{2} S_{5}(1)^{15} S_{4}(1)^{9 / 2}\left\{2 S_{3}(1)^{-3} S_{2}(1 / 2)\right\}^{-3 / 4} \\
& =2^{-7 / 8} S_{6}(1)^{2} S_{5}(1)^{15} S_{4}(1)^{27 / 4} .
\end{aligned}
$$

Applying (2.5) we obtain (4) of Theorem 3. 
Proof of Theorem 4. Using the homogeneity (see [KuKo] for details)

$$
S_{r}\left(c x,\left(c \omega_{1}, \ldots, c \omega_{r}\right)\right)=S_{r}\left(x,\left(\omega_{1}, \ldots, \omega_{r}\right)\right)
$$

and the construction of $S_{2 m}$ we obtain

$$
S_{2 m}\left(m,\left(\underline{1}_{m}, \underline{\tau}_{m}\right)\right)=S_{2 m}\left(m / \tau,\left(\underline{1}_{m}, \underline{\tau^{-1}} m\right)\right)=S_{2 m}\left(m,\left(\underline{1}_{m}, \underline{\tau^{-1}}\right)\right)^{-1} \text {. }
$$

3. Proofs of Theorems 5-8. First, we show Theorems 6-8. We express certain special values of double sine functions via products of usual sine or cosine functions.

LEMMA 3.1.

(1) Let $n$ be a positive integer. Then

$$
\begin{gathered}
S_{2}\left(\frac{1}{2}+n \tau,(1, \tau)\right)=\sqrt{2} \prod_{l=0}^{n}(2 \cos (l \pi \tau))^{-1}, \\
S_{2}\left(\frac{1+\tau}{2}+n \tau,(1, \tau)\right)=\prod_{\substack{l=1 \\
l: \text { odd }}}^{2 n-1}\left(2 \cos \left(\frac{l \pi}{2} \tau\right)\right)^{-1}
\end{gathered}
$$

and

$$
S_{2}\left(\frac{1+\tau}{2}+n,(1, \tau)\right)=\prod_{\substack{l=1 \\ l: \text { odd }}}^{2 n-1}\left(2 \cos \left(\frac{l \pi}{2 \tau}\right)\right)^{-1} .
$$

(2) Let $n \geq 1$ and $N \geq 2$ be integers. Then

$$
\prod_{k=1}^{N-1} S_{2}\left(\frac{k}{N}+n,(1, \tau)\right)=\sqrt{N} \prod_{k=1}^{N-1} \prod_{l=0}^{n-1}\left(2 \sin \left(\frac{\pi}{\tau}\left(\frac{k}{N}+l\right)\right)\right)^{-1} .
$$

(3) Let $n_{1}, n_{2}$ be positive integers. Then

$$
\begin{aligned}
S_{2}\left(\frac{1+\tau}{2}+n_{1}+n_{2} \tau,(1, \tau)\right)= & (-1)^{n_{1} n_{2}} \prod_{\substack{l_{1}=1 \\
l_{1}: \text { odd }}}^{2 n_{1}-1}\left(2 \cos \left(\frac{l_{1} \pi}{2} \frac{1}{\tau}\right)\right)^{-1} \\
& \times \prod_{\substack{l_{2}=1 \\
l_{2}: \text { odd }}}^{2 n_{2}-1}\left(2 \cos \left(\frac{l_{2} \pi}{2} \tau\right)\right)^{-1}
\end{aligned}
$$

Proof. From the quasi-periodicity of multiple sine functions we have

$$
S_{2}\left(\frac{1}{2}+n \tau,(1, \tau)\right)=S_{2}\left(\frac{1}{2},(1, \tau)\right) \prod_{l=0}^{n-1} S_{1}\left(\frac{1}{2}+l \tau\right)^{-1}
$$




$$
\begin{aligned}
S_{2}\left(\frac{1+\tau}{2}+n \tau,(1, \tau)\right)= & \prod_{l=0}^{n-1} S_{1}\left(\frac{1+\tau}{2}+l \tau\right)^{-1} \\
S_{2}\left(\frac{k}{N}+n,(1, \tau)\right)= & S_{2}\left(\frac{k}{N},(1, \tau)\right) \prod_{l=0}^{n-1} S_{1}\left(\frac{k}{N}+l,(\tau)\right)^{-1}, \\
S_{2}\left(\frac{1+\tau}{2}+n_{1}+n_{2} \tau,(1, \tau)\right)= & \prod_{l_{1}=0}^{n_{1}-1} S_{1}\left(\frac{1+\tau}{2}+l_{1},(\tau)\right)^{-1} \\
& \times \prod_{l_{2}=0}^{n_{2}-1} S_{1}\left(\frac{1+\tau}{2}+n_{1}+l_{2} \tau\right)^{-1},
\end{aligned}
$$

where we used

$$
S_{2}\left(\frac{1+\tau}{2},(1, \tau)\right)=1 .
$$

Since $S_{1}(x)=2 \sin (\pi x)$, which is obtained from Lerch's formula [L], and by the homogeneity of multiple sine functions we obtain

$$
\begin{aligned}
S_{1}\left(\frac{1}{2}+l \tau\right) & =2 \cos (l \pi \tau) \\
S_{1}\left(\frac{1+\tau}{2}+l \tau\right) & =2 \cos \left(\frac{(2 l+1) \pi}{2} \tau\right) \\
S_{1}\left(\frac{k}{N}+l,(\tau)\right) & =2 \sin \left(\frac{\pi}{\tau}\left(\frac{k}{N}+l\right)\right) \\
S_{1}\left(\frac{1+\tau}{2}+l_{1},(\tau)\right) & =2 \cos \left(\frac{\left(2 l_{1}+1\right) \pi}{2 \tau}\right), \\
S_{1}\left(\frac{1+\tau}{2}+n_{1}+l_{2} \tau\right) & =(-1)^{n_{1}} 2 \cos \left(\frac{\left(2 l_{2}+1\right) \pi}{2} \tau\right) .
\end{aligned}
$$

Hence (3) of Lemma 3.1 is proved.

Now we show

$$
\prod_{k=1}^{N-1} S_{2}\left(\frac{k \omega_{1}}{N},\left(\omega_{1}, \omega_{2}\right)\right)=\sqrt{N}
$$

In fact, by the definition of $S_{2}$ we have

$$
S_{2}\left(x,\left(\omega_{1}, \omega_{2}\right)\right) S_{2}\left(\omega_{1}+\omega_{2}-x,\left(\omega_{1}, \omega_{2}\right)\right)=1 .
$$

Let $x=k \omega_{1} / N$. Then

$$
\begin{aligned}
\prod_{k=1}^{N-1} S_{2}\left(\frac{k \omega_{1}}{N},\left(\omega_{1}, \omega_{2}\right)\right) \prod_{k=1}^{N-1} S_{2}\left(\omega_{1}-\frac{k \omega_{1}}{N},\left(\omega_{1}, \omega_{2}\right)\right) & \\
& =\prod_{k=1}^{N-1} S_{1}\left(\omega_{1}-\frac{k \omega_{1}}{N},\left(\omega_{1}\right)\right)
\end{aligned}
$$


This gives

$$
\left(\prod_{k=1}^{N-1} S_{2}\left(\frac{k \omega_{1}}{N},\left(\omega_{1}, \omega_{2}\right)\right)\right)^{2}=\prod_{k=1}^{N-1} 2 \sin \left(\frac{k}{N} \pi\right)=N .
$$

Hence (2) of Lemma 3.1 is proved.

When $N=2$, we have

$$
S_{2}\left(\omega_{1} / 2,\left(\omega_{1}, \omega_{2}\right)\right)=\sqrt{2},
$$

which was obtained by Koyama and Kurokawa [KoKu, Theorem 1(2)]. Moreover, from the homogeneity of multiple sine functions and

$$
S_{2}\left(\frac{1+\tau}{2}+n \tau,(1, \tau)\right)=\prod_{\substack{l=1 \\ l: \text { odd }}}^{2 n-1}\left(2 \cos \left(\frac{l \pi}{2} \tau\right)\right)^{-1},
$$

we have

$$
S_{2}\left(\frac{1+\tau}{2}+n,(1, \tau)\right)=\prod_{\substack{l=1 \\ l: \text { odd }}}^{2 n-1}\left(2 \cos \left(\frac{l \pi}{2 \tau}\right)\right)^{-1} .
$$

Thus, we obtain (1) of Lemma 3.1.

LEMMA 3.2.

(1) Let $n \geq 2$ be an integer and $\tau \in \overline{\mathbb{Q}}-\mathbb{Q}$. Then

$$
\prod_{l=0}^{n-1} \cos (l \pi \tau) \notin \overline{\mathbb{Q}} \text {. }
$$

(2) Let $n$ be a positive integer and $\tau \in \overline{\mathbb{Q}}-\mathbb{Q}$. Then

$$
\prod_{\substack{l=1 \\ l: \text { odd }}}^{2 n-1} \cos \left(\frac{l \pi}{2} \tau\right) \notin \overline{\mathbb{Q}} \quad \text { and } \quad \prod_{\substack{l=1 \\ l: \text { odd }}}^{2 n-1} \cos \left(\frac{l \pi}{2 \tau}\right) \notin \overline{\mathbb{Q}} .
$$

(3) Let $n \geq 1$ and $N \geq 2$ be integers and $\tau \in \overline{\mathbb{Q}}-\mathbb{Q}$. Then

$$
\prod_{k=1}^{N-1} \prod_{l=0}^{n-1} \sin \left(\frac{\pi}{\tau}\left(\frac{k}{N}+l\right)\right) \notin \overline{\mathbb{Q}} \text {. }
$$

(4) Let $n_{1}, n_{2}$ be positive integers and $\tau \neq 0$ be quadratic irrational. Then

$$
\prod_{\substack{l_{1}=1 \\ l_{1}: \text { odd }}}^{2 n_{1}-1} \cos \left(\frac{l_{1} \pi}{2} \frac{1}{\tau}\right) \prod_{\substack{l_{2}=1 \\ l_{2}: \text { odd }}}^{2 n_{2}-1} \cos \left(\frac{l_{2} \pi}{2} \tau\right) \notin \overline{\mathbb{Q}}
$$


Proof. First we prove (1). Suppose otherwise. Put $q=e^{\pi i \tau}$. Then

$$
\prod_{l=0}^{n-1}\left(q^{l}+q^{-l}\right) \in \overline{\mathbb{Q}}
$$

Hence there is a $\overline{\mathbb{Q}}$-algebraic equation for $q$. However, the Gelfond-Schneider theorem (see [Bak, Theorem 2.1]) gives $q=(-1)^{\tau} \notin \overline{\mathbb{Q}}$. The contradiction proves (1).

Next we prove (2). Suppose otherwise. Put $q=e^{\frac{\pi i}{2} \tau}$ or $q=e^{\frac{\pi i}{2 \tau}}$. Then

$$
\prod_{\substack{l=1 \\ l: \text { odd }}}^{2 n-1}\left(q^{l}+q^{-l}\right) \in \overline{\mathbb{Q}} .
$$

Similarly, this gives a contradiction.

Next we prove (3). Suppose otherwise. Put $q=e^{\frac{\pi i}{N \tau}}$. Then

$$
\prod_{k=1}^{N-1} \prod_{l=0}^{n-1}\left(q^{k+l N}-q^{-(k+l N)}\right) \in \overline{\mathbb{Q}}
$$

Similarly, this gives a contradiction.

Finally, we prove (4). Since $\tau$ is a quadratic irrational number, there are integers $N, M$ such that $\tau=\frac{N}{M} \frac{1}{\tau}$. Suppose otherwise. Put $q=e^{\frac{\pi i}{2 M \tau}}$. Then

$$
\prod_{\substack{l_{1}=1 \\ l_{1} \text { :odd }}}^{2 n_{1}-1}\left(q^{M l_{1}}+q^{-M l_{1}}\right) \prod_{\substack{l_{2}=1 \\ l_{2} \text { :odd }}}^{2 n_{2}-1}\left(q^{N l_{2}}+q^{-N l_{2}}\right) \in \overline{\mathbb{Q}} .
$$

Similarly, this gives a contradiction.

Proofs of Theorems 6 and \%. The theorems follow from Lemmas 3.1 and 3.2 .

Proof of Theorem 8. When $\alpha$ is rational, $\sin (\alpha \pi), \cos (\alpha \pi) \in \overline{\mathbb{Q}}$. Hence Theorem 8 follows.

Proof of Theorem 5. We prove (1) of Theorem 5 by induction on $r=$ $2,3, \ldots$ When $r=2$, from the result of Kurokawa and Wakayama [KW, Theorem 1.2] we see that $S_{2}(2,(1, \tau))$, is transcendental. Assume that one of the numbers $S_{r}\left(t+1,\left(\underline{1}_{r-1}, \tau\right)\right), t=1, \ldots, r-1$ is transcendental. Then from the quasi-periodicity of multiple sine functions for $k=3, \ldots, r+1$ we have

$$
S_{r+1}\left(k,\left(\underline{1}_{r}, \tau\right)\right) S_{r+1}\left(k-1,\left(\underline{1}_{r}, \tau\right)\right)^{-1}=S_{r}\left(k-1,\left(\underline{1}_{r-1}, \tau\right)\right)^{-1} .
$$

So, one of the $r$ numbers $S_{r+1}\left(t+1,\left(\underline{1}_{r}, \tau\right)\right), t=1, \ldots, r$, is transcendental. Similarly, from Theorem 6(1) \& (2) and Theorem 7 we have (2), (3) and (4) of Theorem 5 respectively. 
Acknowledgements. The author expresses his sincere thanks to Professor Nobushige Kurokawa, who gave him helpful advice. The author also thanks the referee for some helpful comments.

\section{References}

[Bak] A. Baker, Transcendental Number Theory, Cambridge Univ. Press, 1975.

[D] C. Deninger, Local L-factors of motives and regularized determinant, Invent. Math. 107 (1992), 135-150.

$[\mathrm{KoKu}]$ S. Koyama and N. Kurokawa, Values of the double sine function, J. Number Theory 123 (2007), 204-223.

[KuKo] N. Kurokawa and S. Koyama, Multiple sine functions, Forum Math. 15 (2003), 839-876.

[KW] N. Kurokawa and M. Wakayama, Algebraicity and transcendency of basic special values of Shintani's double sine functions, Proc. Edinburgh Math. Soc. 49 (2006), 361-366.

[L] M. Lerch, Further studies in the domain of Malmstén's series, Rozpravy České Akad. 3 (1894), no. 28, 61 pp. (in Czech).

[S] T. Shintani, On a Kronecker limit formula for real quadratic fields, J. Fac. Sci. Univ. Tokyo Ser. IA Math. 24 (1977), 167-199.

[Tana] H. Tanaka, Special values of multiple sine functions, Kyushu J. Math. 62 (2008), 123-137.

[Tang] B. Tangedal, Continued fractions, special values of the double sine function, and Stark units over real quadratic fields, J. Number Theory 124 (2007), 291-313.

Department of Mathematics

Tokyo Institute of Technology

Meguro, Tokyo 152-8551, Japan

E-mail: h.tanaka@math.titech.ac.jp

Received on 7.5.2008

and in revised form on 17.8.2008 